Journal of Animal and Veterinary Advances 10 (16): 2120-2126, 2011

ISSN: $1680-5593$

(C) Medwell Journals, 2011

\title{
Development and Application of the Novel Visual Loop-Mediated Isothermal Amplification of Omp25 Sequence for Rapid Detection of Brucella sp.
}

\author{
Wen Pan, Jia-Ying Wang, Hai-Yan Shen, Ming-Qiu Zhao, Chun-Mei Ju, \\ Xiao-Ying Dong, Lin Yi and Jin-Ding Chen \\ College of Veterinary Medicine, South China Agricultural University, \\ $483 \mathrm{Wu}$ Shan Road, Tianhe District, 510640 Guangzhou, P.R. China
}

\begin{abstract}
A novel visual Loop-mediated isothermal Amplification (LAMP) method for detecting Brucella sp. was established. LAMP primers were designed by targeting the species-specific omp 25 gene. In this LAMP reaction, manganous ion and calcein were added which allows the results to be simply judged by naked eye under daylight by color change from orange to green in a closed-tube system without expensive equipment and it took $<60 \mathrm{~min}$ at $63^{\circ} \mathrm{C}$. The assay correctly identified 3 Brucella $\mathrm{sp}$. strains but did not detect 8 other bacterial species. Detection limit of genomic DNA of Brucella sp. was $10 \mathrm{pg}$. The assay was also successfully applied for the detection of Brucella sp. from contaminated milk (as low as $1.3 \times 10^{3} \mathrm{CFU} \mathrm{mL}^{-1}$ ) and infected mice tissues. The assay shows enormous potential for rapidly, expediently and accurately detecting Brucella sp. from food and clinical samples.
\end{abstract}

Key words: Brucella sp., visual LAMP, omp25 gene, detection, food, China

\section{INTRODUCTION}

Brucella sp., the causative agents of brucellosis are pathogenic to a variety of domestic and wild animals as well as humans. Brucella abortus, B. suis and $B$. melitensis are listed as category B biothreat agents by the Centers for Disease Control and Prevention Strategic Planning Group (Rotz et al., 2002). Brucellosis, a major zoonotic disease worldwide which impacts public health and agricultural economic problems in many countries is acquired by humans primarily through contact with infected livestock and consumption of unpasteurized dairy products.

Due to the complexity of disease presentation, the diagnosis of brucellosis is currently based on bacteriological isolation, serological tests (Baum et al., 1995; Nielsen, 2002). However, these tests mentioned above are time-consuming, require skilled technicians and are not straightforward. Although, DNA-based methods such as PCR have been widely used for diagnosing brucellosis (Herman and de Ridder, 1992; Leal-Klevezas et al., 1995), they are not appropriate for resource-limited laboratories since costly specialized equipments are needed. Therefore, the development of rapid, accurate, sensitive, cost-effective and more practical diagnostic methods for diagnosis of brucellosis and identification of Brucella sp. are of high priority.
Loop-Mediated isothermal Amplification (LAMP) is an outstanding nucleic acid amplification method which relies on autocycling strand displacement DNA synthesis performed by the Bst DNA polymerase large fragment (Notomi et al., 2000). More than 180 reports evaluating the LAMP method have been published since the assay was originally developed. After amplification, the products generated by LAMP could be either examined by gel electrophoresis generating a ladder of DNA fragments or simply detected visually fluorescence by adding fluorescent dyes to the reaction tube after amplification (Iwamoto et al., 2003; Qiao et al., 2007).

However, the detection of the conventional LAMP product mentioned above all have their own shortcomings. The most serious problem is a high risk of workspace contamination with an extremely large amount of amplicon when opening the reaction tube. Therefore, a closed reaction system for easy readout of LAMP result needs to be developed. Recently, Tomita et al. (2008) reported an improved LAMP by adding calcein and manganous ion, a fluorescent metal indicator to the reaction solution before amplification so that the result could be directly evaluated by visual observation of color change of the solution from orange to green without opening tube or detection device. Additionally the novel visual LAMP has been successfully applied to detection of Mycobacterium tuberculosis and Mycobacterium bovis (Zhu et al., 2009).

Corresponding Author: W. Pan, College of Veterinary Medicine, South China Agricultural University, 483 Wu Shan Road, Tianhe District, 510640 Guangzhou, P.R. China 
In this study, we aimed to establish a novel visual LAMP method based on the conventional LAMP method for the detection of Brucella sp. with which the result can be discriminate more easily and clearly. As compared with the conventional LAMP assay and PCR method, the sensitivity, specificity and applicability of the novel visual LAMP were evaluated. These advantages of the novel visual LAMP make it a promising platform for the development of a more economical and practical tool for the molecular detection of Brucella sp. in resource-limited laboratories as well as the field condition.

\section{MATERIALS AND METHODS}

Bacterial strains: Brucella abortus strain S19, Brucella melitensis strain M5, Brucella suis strain S2 were purchased from China Institute of Veterinary Drug Control. The 8 other commonly encountered bacterial species used to determine the specificity of LAMP were deposited in College of Veterinary Medicine, South China Agricultural University including Escherichia coli, Salmonella typhimurium, Staphylococcus aureus, Pasteurella multocida, Streptococcus pneumonia, Sarcina lutea, Bacillus subtilis and Legionella pneumophila. All bacterial pathogens were handled in BSL3 or BSL2 rooms recognized by the Safety Control Committee of South China Agricultural University.

Extraction of genomic DNA: The DNA of bacteria was extracted according to the modified $\mathrm{CTAB} / \mathrm{NaCl}$ protocol (Romero and Lopez-Goni, 1999). Briefly, brucella strains were incubated in $3 \mathrm{~mL}$ of tryptic soy agar (Oxoid Ltd., London, England) supplemented with yeast extract (Oxoid Ltd., London, England) for $24 \mathrm{~h}$ at $37^{\circ} \mathrm{C}$. The $1 \mathrm{~mL}$ of the suspension was taken and centrifuged at $6,000 \times \mathrm{g}$ for $10 \mathrm{~min}$. The cell pellets were resuspended with $200 \mu \mathrm{L}$ NET buffer (50 mMNaCl, $125 \mathrm{mMEDTA}, 50 \mathrm{mMTris}-\mathrm{HCl}$ [pH7.6]). After incubation at $80^{\circ} \mathrm{C}$ for $10 \mathrm{~min}$ with $100 \mu \mathrm{L}$ $10 \%$ of Sodium Dodecyl Sulfate (SDS), the mixture was cooled on ice for $2 \mathrm{~min}$ and digested with RNase $\left(100 \mathrm{mg} \mathrm{mL}^{-1}\right)$ and proteinase $\mathrm{K}\left(200 \mathrm{mg} \mathrm{mL}^{-1}\right)$ at $56^{\circ} \mathrm{C}$ for $1 \mathrm{~h}$. Cell debris was removed by precipitation with $5 \mathrm{M}$ $\mathrm{NaCl}$ and a hexadecyltrimethylammonium bromide- $\mathrm{NaCl}$ (CTAB-NaCl) solution at $65^{\circ} \mathrm{C}$ for $10 \mathrm{~min}$. DNA was extracted by the standard protocol with phenolchloroform-isoamyl alcohol, precipitated with isopropanol, washed with $70 \%$ ethanol and dried under a vacuum. The DNA pellet was dissolved in $25 \mu \mathrm{L}$ of sterile distilled water and stored at $-20^{\circ} \mathrm{C}$ for further use.

Primer design: Four Brucella-specific primers for LAMP were designed based on the species-specific omp 25 gene (GeneID: X79284.1) sequence following the criteria described previously (Notomi et al., 2000; Tomita et al.,

\begin{tabular}{llrc}
\multicolumn{5}{l}{ Table 1: Nucleotide sequences for the visual LAMP primers } \\
\hline Primer type & Sequence (5'-3') & Length & Target \\
\hline F3 & AAGTCTCTCGTAATCGTCTCGG & 22 & omp 25 gene \\
B3 & CCAGCCTTCCAATCGTCAG & 19 & omp25 gene \\
FIP (F1c+F2) & TACTGGGGAGCTACTTCAACC & 43 & omp25 gene \\
& GGATCTCTGCGACCGCTTTTGC & & \\
BIP (B1c+B2) & GCTGGGCTGGTGGCTATACCGT & 40 & omp25 gene \\
& GATGCTGCCAACGGTGCT & & \\
\hline
\end{tabular}

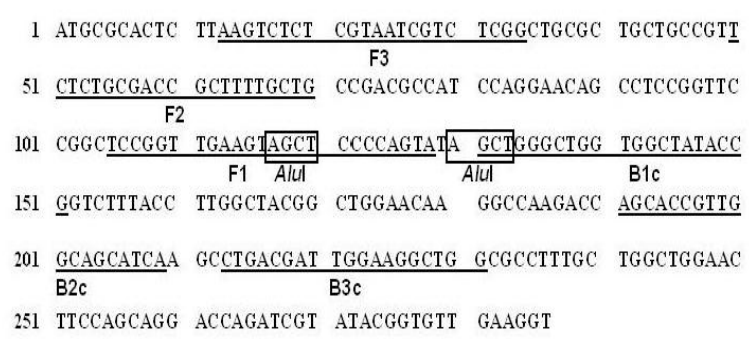

Fig. 1: Nucleotide sequence of omp25 gene of Brucella abortus (GeneID: X79284.1) used for designing the primers. Locations of target sequences of primers are underlined and the AluI restriction site is indicated by box

2008 ) including two outer primers (F3 and B3) and two inner primers (FIP and BIP) (Table 1). These primers recognize six distinct regions on the target DNA (Fig. 1). The Forward Inner Primer (FIP) is comprised of F1c sequence complementary to F1 and F2 sequence. The Backward Inner Primer (BIP) is comprised of B1c sequence complementary to $\mathrm{B} 1$ and $\mathrm{B} 2$ sequence.

Optimization of the novel visual lamp condition: The novel visual LAMP assay was performed by adding calcein and manganese chloride based on the conventional LAMP. The final $25 \mu \mathrm{L}$ reaction mixture consisted of $1.6 \mu \mathrm{M}$ (each) of FIP and BIP primers, $0.4 \mu \mathrm{M}$ (each) of each F3 and B3 outer primers, $400 \mu \mathrm{M}$ of each dNTP, $1 \mathrm{M}$ betaine (Sigma, St. Louis, MO), $25 \mu \mathrm{M}$ calcein (Sigma), $0.5 \mathrm{mM} \mathrm{MnCl}$ (Sigma), $2 \mathrm{mM} \mathrm{MgSO}_{4}$, $1 \times$ thermopol buffer (New England Biolabs, Beverly, MA, USA), $8 \mathrm{U}$ of Bst DNA polymerase large fragment (New England Biolabs, Beverly, MA, USA) and $1 \mu \mathrm{L}$ of genomic DNA (10 ng). The optimal response time and temperature was maintained at $63^{\circ} \mathrm{C}$ for $60 \mathrm{~min}$. The reaction was terminated by heating to $95^{\circ} \mathrm{C}$ for $2 \mathrm{~min}$.

PCR reaction: $P C R$ reaction was carried out with $F 3$ and B3 primers in $25 \mu \mathrm{L}$ volume reaction mixture containing $2.5 \mu \mathrm{L} 10 \times$ Ex Taq buffer, $0.2 \mathrm{mM}$ of each dNTP, $0.2 \mu \mathrm{M}$ of each primer and $0.625 \mathrm{U}$ TaKaRa Ex Taq DNA polymerase (TaKaRa, DaLian, China) and $1 \mu \mathrm{L}$ of template DNA. The cycling conditions consisted of initial denaturation at $95^{\circ} \mathrm{C}$ for $5 \mathrm{~min}$ followed by 30 cycles of denaturation at $95^{\circ} \mathrm{C}$ for $30 \mathrm{sec}$, annealing at $55^{\circ} \mathrm{C}$ for 30 $\mathrm{sec}$ and extension at $72^{\circ} \mathrm{C}$ for $45 \mathrm{sec}$ and a final extension at $72^{\circ} \mathrm{C}$ for $10 \mathrm{~min}$. 
Detection and analysis of LAMP and PCR products: After amplification, the results of the novel visual LAMP reaction could be simply judged by naked eye under daylight or UV light. The $5 \mu \mathrm{L}$ of LAMP and PCR amplified products were electrophoresed in $2 \%$ agarose gel stained with ethidium bromide, respectively. To confirm the structures of the LAMP amplified products, the amplicons were digested with restriction enzyme AluI (TaKaRa, DaLian, China) at $37^{\circ} \mathrm{C}$ for $3 \mathrm{~h}$. The target PCR products were cloned into pGEM-T Easy Vector (Promega, WI, USA) and sequenced by using an ABI Prism 3730 DNA sequencer (Perkin-Elmer, Wellesley, MA, USA).

Specificity and sensitivity of the novel visual LAMP: Specificity and sensitivity of the LAMP and PCR method were all carried out under optimal reaction condition. To evaluate the specificity of the LAMP primers, 3 Brucella sp. strains and 8 other bacterial species were tested. To study the detection limit of the novel visual LAMP assay, serial 10 fold dilutions of purified genomic DNA of Brucella abortus were tested and the results were compared with those of the conventional LAMP assay and PCR method. The initial amount of purified DNA was $100 \mathrm{ng}$. The results of the novel visual LAMP assay were analysed through visual detection under day light and UV light and accurately confirmed by gel electrophoresis. The detection limit was defined as the last positive dilution and the reactions were done in triplicates. The sterile water was used as negative control instead of the DNA temple in each reaction cycle.

Evaluation of the novel visual LAMP assay for artificially contaminated milk and infected mice tissues with $B$. abortus S19 strain: The $100 \mu \mathrm{L} 10$ fold serial dilutions of B. abortus $\mathrm{S} 19$ were added into $900 \mu \mathrm{L}$ milk, respectively and the final concentrations were in the range of $1.3 \times 10^{7}-1.3 \times 10^{0} \mathrm{CFU} \mathrm{mL}^{-1}$. At the same time, one sample of sterile milk was used as internal negative control. DNA was extracted by the same method as described above.

SPF KM Mice (4-6 weeks old) were injected intraperitoneally with $5.2 \times 10^{9}$ cells of B. abortus S19 strain and sacrifced 10 days after injection by cervical dislocation under ether anaesthesia. The spleen and liver were isolated and used for DNA extraction and enumeration of bacteria in organs.

DNA of livers ( $25 \mathrm{mg}$ each) and spleens (10 mg each) from infected mice were extracted by the same method as described above. Meanwhile DNA extracted from spleen and liver of SPF KM Mice without infected were used as internal negative control, respectively. For enumeration of bacteria, spleens and liver (100 mg each) were grinded in $1 \mathrm{~mL}$ of sterile physiological saline respectively and mixed well. About $500 \mu \mathrm{L}$ mixture was added into $4.5 \mathrm{~mL}$ sterile physiological saline, serial 10 fold diluted, plated appropriate dilutions on TSB-YE agar at $37^{\circ} \mathrm{C}$ with $5 \% \mathrm{CO}_{2}$ for $72 \mathrm{~h}$ and enumerated the number of bacteria.

The novel visual LAMP reaction was carried out under optimal amplified conditions using $1 \mu \mathrm{L}$ of the extracted DNA and done in triplicates. Sterile doubledistilled water was used as negative control.

\section{RESULTS AND DISCUSSION}

Establishment of the novel visual LAMP assay for Brucella sp.: Result of the novel visual LAMP reaction could be simply observed by naked eye under day light or UV light (Fig. 2). Under day light, the positive reaction (with target DNA) turned green while the negative reaction (without target DNA) remained orange; under UV light, the positive reaction emitted strong green fluorescence while the negative reaction did not emit any fluorescence.

Analysis of LAMP and PCR products: The characteristic ladder-like pattern of bands was confirmed by agarose gel electrophoresis (Fig. 3). The amplification products were digested by restriction enzyme AluI and the electrophoresis of digestion products generated a strong band at approximately $100 \mathrm{bp}$, corresponding to the three similar theoretically predicted sizes $(98,100$ and $113 \mathrm{bp})$ (Fig. 3). PCR products were analysed by gel electrophoresis and generated a single band of $218 \mathrm{bp}$ in accord with expected size. The cloned sequence was $100 \%$ identical with the target sequence of omp 25 published in the Gene Bank.

Specificity and sensitivity of the LAMP: The primers of LAMP correctly identified 3 Brucella sp. strains while no amplification products were detected from any of the other bacterial species and negative control. The results were showed via electrophoresis (Fig. 4). The detection
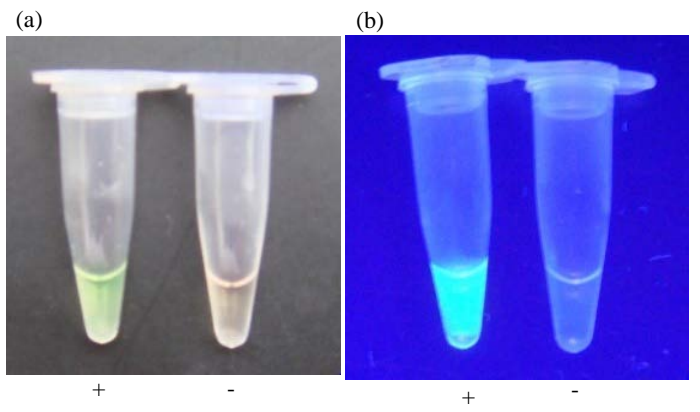

Fig. 2: Visual detection of omp25 LAMP reaction using fluorescent metal indicator. (a) Under daylight; (b) Under UV light; plus sign denotes positive reaction (with target DNA); minus sign denotes negative reaction (without target DNA) 


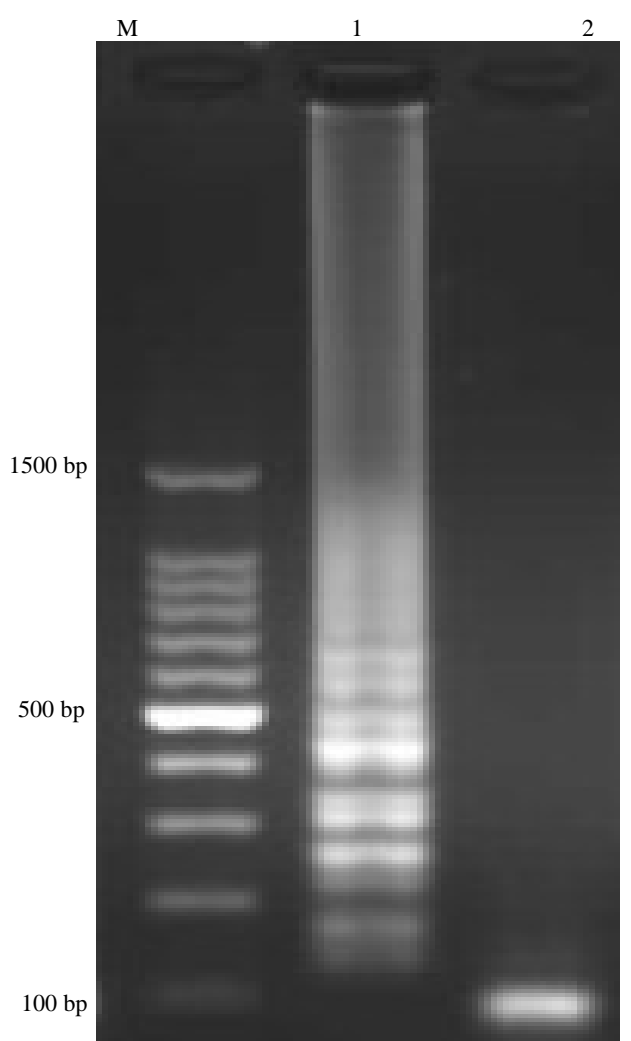

Fig. 3: Agarose gel electrophoresis analysis of LAMP reaction products and the digestion result of AluI restriction enzyme. Lane 1: Agarose gel electrophoresis results for LAMP reaction products; Lane 2: The digestion products of LAMP product after AluI restriction enzyme digestion; M: 100 bp marker

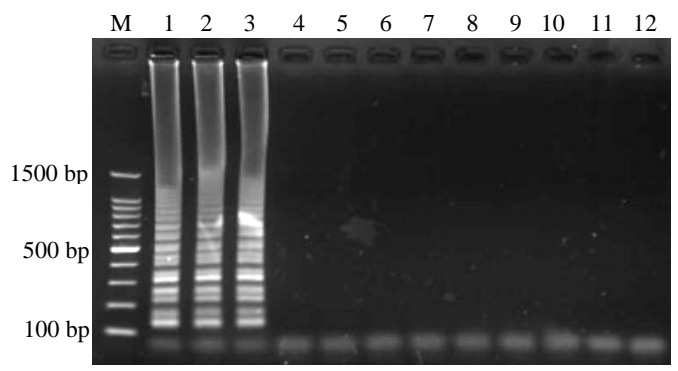

Fig. 4: Specificity of the LAMP assay among various bacterial DNAs, Lane 1: B. abortus S19; Lane 2: $B$. suis S2; Lane 3: B. melitensis M5; Lane 4: Escherichia coli; Lane 5: Salmonella typhimurium; Lane 6: Staphylococcus aureus; Lane 7: Pasteurella multocida, Lane 8: Streptococcus pneumonia; Lane 9: Sarcina lutea; Lane 10: Bacillus subtilis; Lane 11: Legionella pneumophila; Lane 12, negative control with sterilized double-distilled water; M: 100 bp marker

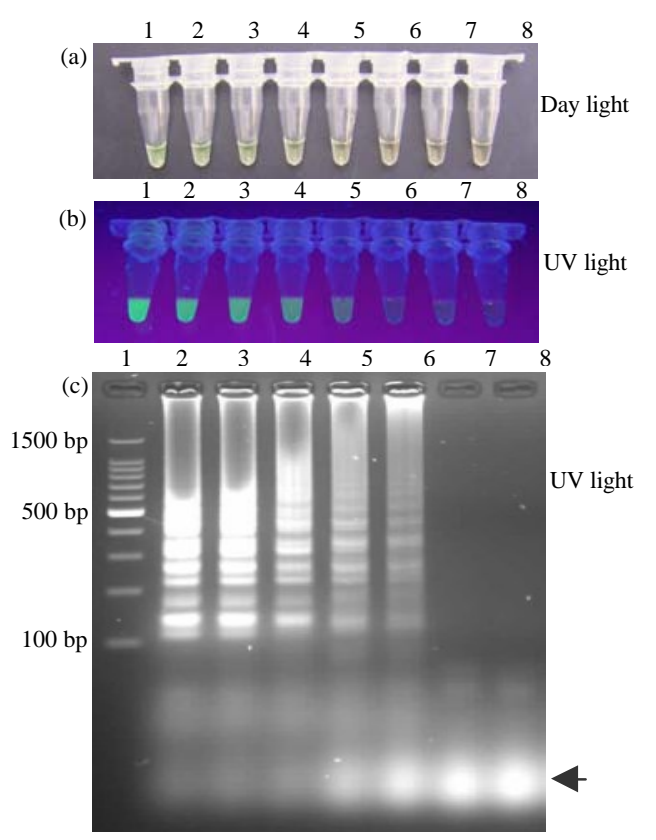

Fig. 5: Analytical sensitivity of the novel visual LAMP. (a) Sensitivity of visual LAMP under under daylight; (b) Sensitivity of visual LAMP under UV light; (c) Agarose gel electrophoresis results for sensitivity of visual LAMP. Lane 1: $100 \mathrm{ng}$; Lane 2: $10 \mathrm{ng}$; Lane 3: $1 \mathrm{ng}$; Lane 4: $100 \mathrm{pg}$; Lane 5: $10 \mathrm{pg}$; Lane 6: $1 \mathrm{pg}$; Lane 7: $100 \mathrm{fg}$; Lane 8: Negative control with sterilized double-distilled water; $\mathrm{M}$ : 100 bp marker

limit of the visual LAMP was $10 \mathrm{pg}$ of genomic DNA of Brucella abortus (Fig. 5) which was consistent with those of the conventional LAMP assay and PCR (Fig. 6).

Sensitivity of the novel visual LAMP for artificially contaminated milk: The detection limit of the novel visual LAMP assay for B. abortus in artificially contaminated milk was as low as $1.3 \times 10^{3} \mathrm{CFU} \mathrm{mL}^{-1}$. If the process of DNA extraction is $100 \%$ efficient, the accomplished sensitivity of $1.3 \times 10^{3} \mathrm{CFU}$ in $1 \mathrm{~mL}$ of milk corresponding to approximately $52 \mathrm{CFU}$ of $B$. abortus per visual LAMP reaction (Fig. 7).

The novel visual LAMP for infected tissues with $B r$. abortus S19 strain: Colony counting of spleens and livers from infected mice with $B$. abortus was $1.2 \times 10^{6}$ and $2.5 \times 10^{5} \mathrm{CFU} / 100 \mathrm{mg}$, respectively. The novel visual LAMP assay applied in infected spleens and livers with $B$. abortus all showed positive resultss while the liver and spleen DNA from uninfected mice show negative results. 

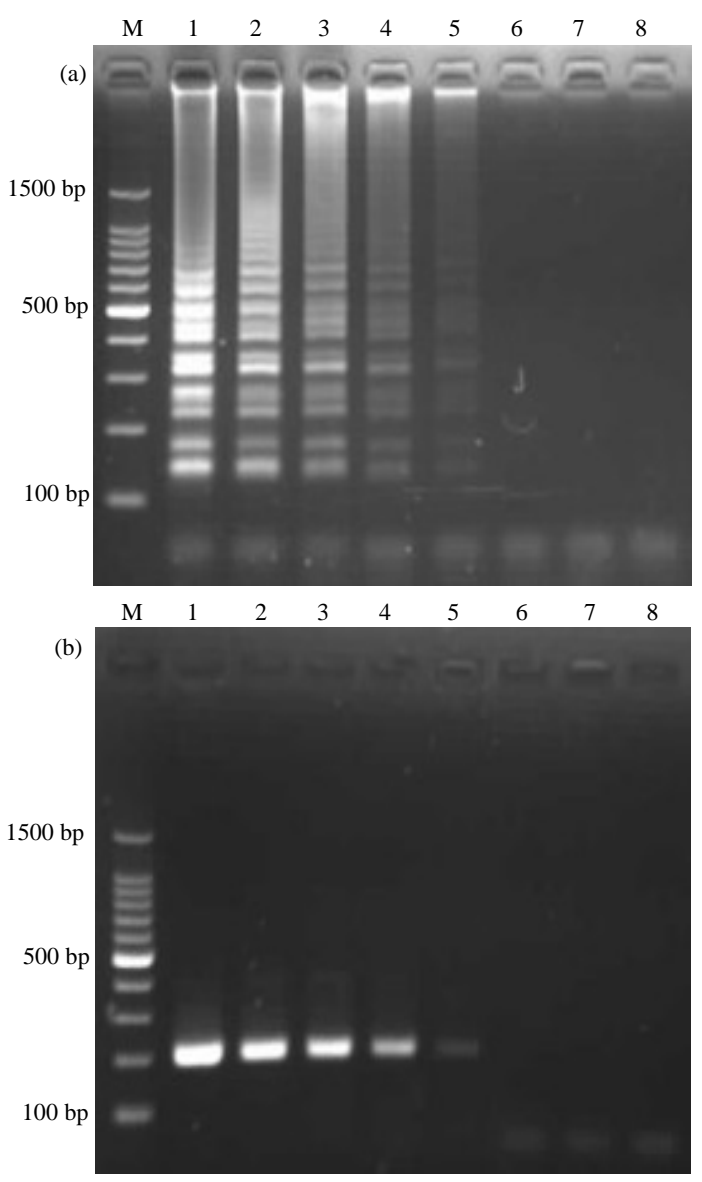

Fig. 6: Agarose gel electrophoresis results for sensitivity of conventional LAMP and PCR. (a) Sensitivity of conventional LAMP (b) Sensitivity of PCR. Lane 1: 100 ng; Lane 2: 10 ng; Lane 3: 1 ng; Lane 4: 100 pg; Lane 5: 10 pg; Lane 6: 1 pg; Lane 7: 100 fg; Lane 8: Negative control with sterilized double-distilled water; $\mathrm{M}: 100 \mathrm{bp}$ marker

Since, brucellosis is a zoonosis, fast and accurate detection of Brucella sp. is very important for treating and preventing or even eradicating brucellosis. This study successfully developed a novel visual LAMP assay for rapid detection of Brucella sp. in contaminated milk and infected tissues which is significant for quarantine inspection of Brucella sp. and monitoring survey of brucellosis.

The most attractive characteristics of the novel visual LAMP assay is the simple and effective endpoint visual detection system which not only simplifies the determination of results with no need for time-consuming electrophoresis and costly specialized equipment but also minimizes the risk of workspace contamination with (a)
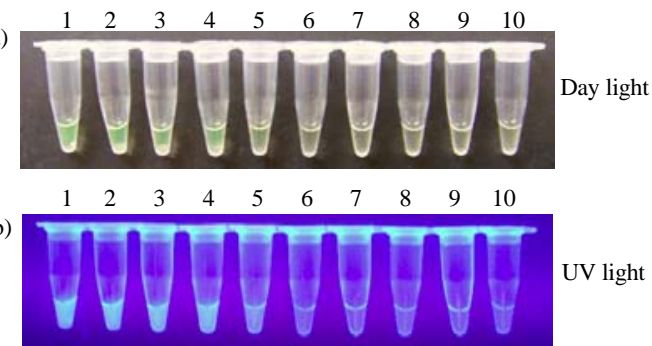

Fig. 7: The sensitivity test of the novel visual LAMP assay from contaminated milk with Brecella abortus. lane 1: $1.3 \times 10^{7} \mathrm{CFU} \mathrm{mL}^{-1}$; Lane $2: 1.3 \times 10^{6}$ CFU $\mathrm{mL}^{-1}$; Lane 3: $1.3 \times 10^{5} \mathrm{CFU} \mathrm{mL}^{-1}$; Lane 4: $1.3 \times 10^{4} \mathrm{CFU} \mathrm{mL}^{-1}$; Lane $5: 1.3 \times 10^{3} \mathrm{CFU} \mathrm{mL}^{-1}$; Lane 6: $1.3 \times 10^{2} \mathrm{CFU} \mathrm{mL}^{-1}$; Lane $7: 1.3 \times 10^{1} \mathrm{CFU} \mathrm{mL}^{-1}$;

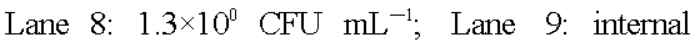
negative control with DNA of sterile milk; Lane 10: Negative control with sterilized double-distilled water; M: 100 bp marker

amplicon. Compared with the real-time LAMP assay (Ohtsuki et al., 2008), the novel visual LAMP assay is more practical without sophisticated real-time thermal cycler. The proportion of calcein and manganese ion concentration is the key of results in the detection system of the novel visual LAMP. If the concentration of calcein is too high, the judgement of results will be interfered with the fuorescence that can not be completely shielded by manganese ions under the UV light. If the concertation of calcein is too low, a large number of manganese ions can not completely form an insoluble salt with the pyrophosphate generated as reaction proceeds and the fluorescence given out by cacein was remain shielded by the remainder of the manganese ions after reaction which result in a longer reaction time and the solution remained orange. The optical concentration of calcein and manganous ion were $25 \mu \mathrm{M}$ and $0.5 \mathrm{mM}$ in $25 \mu \mathrm{L}$ reaction mixture through screening. The simplicity and clarity of the result detection system should make it applicable even for resource-limited laboratories in rural areas of developing countries.

The highly specificity of the LAMP mainly attributed to four specially LAMP primers recognizing six distinct regions on the omp 25 gene sequence which was highly conserved in Brucella species, biovars and strains (Cloeckaert et al., 1995, 1996). The assay successfully identified 3 Brucella sp. strains but 8 other bacterial species could not be detected. The sizes of the fragments generated from the LAMP reaction and AluI restriction enzyme digestion were in accordance with the sizes predicted theoretically, demonstrating that the amplified products were unique and specific. 
In this study, the detection limit of the novel visual LAMP was $10 \mathrm{pg}$ of genomic DNA per tube with similar to those of the conventional LAMP and PCR. In most references the detection limit of the LAMP was estimated to be $0.5-5$ pg of DNA per tube (Enosawa et al., 2003; Yamazaki et al., 2008) which was greater than that for conventional PCR. Designing appropriate primers for LAMP was one of the most important key points in optimizing the LAMP reaction, different sets of primers indicated different sensitivity levels (Enosawa et al., 2003; Ohtsuki et al., 2008; Sakuma et al., 2009). So, examination and further improvement of the primers might improve the sensitivity of detection of LAMP in the future. Because the natural infection samples of brucellosis were few and difficult to obtain, the samples from experimental infected SPF KM mice with $B$. abortus S19 strain were detected. The novel visual LAMP assay was also successfully applied for the detection of Brucella sp. in artificially infected mice tissues and contaminated milk.

In consideration of the extensive application of the novel visual LAMP assay in this assay genomic DNA from cultured cells, artificial contaminated milk and infected tissues was extracted using the modified $\mathrm{CTAB} / \mathrm{NaCl}$ protocol (Romero and Lopez-Goni, 1999) which was relatively cheap and also could be employed in resource-limited laboratories in developing countries.

\section{CONCLUSION}

In the study, the novel visual LAMP assay has an advantage over the conventional biochemical, PCR and the conventional LAMP methods due to its rapid reaction, simple, economical and practical operation, endpoint visual detection system with minimal contamination. It indicates that the novel visual LAMP can be a convenient molecular diagnostic tool for for rapid detection of Brucella sp. from food and clinical samples and surveillance of brucellosis.

\section{ACKNOWLEDGEMENTS}

This research was supported by grants from Guangdong animal and plant epidemic prevention and quarantine research programs (guangdong finance and agriculture [2009] 225), the joint project of the National Natural Science Foundation of China and the Guangdong Natural Science Foundation (no. U0631006), the Program for Changjiang Scholars and Innovative Research Teams in Chinese Universities (no. IRT0723), the Program for Innovative Research Teams of Guangdong Natural Science Fou;ndation (no.5200638).
This research is supported by Special Fund for Agro-Scientific Research in the public Interest (no. 201203056).

\section{REFERENCES}

Baum, M., O. Zamir, R. Bergman-Rios, E. Katz, Z. Beider, A. Cohen and M. Banai, 1995. Comparative evaluation of microagglutination test and serum agglutination test as supplementary diagnostic methods for brucellosis. J. Clin. Microbiol., 33: 2166-2170.

Cloeckaert, A., J.M. Verger, M. Grayon and N. Vizcaino, 1996. Molecular and immunological characterization of the major outer membrane proteins of Brucella. FEMS Microbiol. Lett., 145: 1-8.

Cloeckaert, A., J.M. Verger, M. Grayon and O. Grepinet, 1995. Restriction site polymorphism of the genes encoding the major $25 \mathrm{kDa}$ and $36 \mathrm{kDa}$ outermembrane proteins of Brucella. Microbiology, 141: 2111-2121.

Enosawa, M., S. Kageyama, K. Sawai, K. Watanabe and T. Notomi, et al., 2003. Use of loop-mediated isothermal amplification of the IS900 sequence for rapid detection of cultured Mycobacterium avium subsp. Paratuberculosis. J. Clin. Microbiol., 1 41: 4359-4365.

Herman, L. and H. de Ridder, 1992. Identification of Brucella spp. by using the polymerase chain reaction. Applied Environ. Microbiol., 58: 2099-2101.

Iwamoto, T., T. Sonobe and K. Hayashi, 2003. Loopmediated isothermal amplification for direct detection of Mycobacterium tuberculosis complex, $M$. avium and $M$. intracellulare in sputum samples. J. Clin. Microbiol., 41: 2616-2622.

Leal-Klevezas, D.S., I.O. Martinez-Vazquez, A. LopezMerino, and J.P. Martinez-Soriano, 1995. Single-step PCR for detection of Brucella spp. from blood and milk of infected animals. J. Clin. Microbiol., 33: 3087-3090.

Nielsen, K., 2002. Diagnosis of brucellosis by serology. Vet. Microbiol., 90: 447-459.

Notomi, T., H. Okayama, H. Masubuchi, T. Yonekawal, K. Watanabe, N. Amino and T. Hase, 2000. Loopmediated isothermal amplification of DNA. Nucl. Acids Res., 28: e63-e63.

Ohtsuki, R., K. Kawamoto, Y. Kato, M.M. Shah, T. Ezaki and S.I. Makino, 2008. Rapid detection of Brucella spp. by the loop-mediated isothermal amplification method. J. Applied Microbiol., 104: 1815-1823. 
Qiao, Y.M., Y.C. Guo, X.E. Zhang, Y.F. Zhou and Z.P. Zhang et al., 2007. Loop-mediated isothermal amplification for rapid detection of Bacillus anthracis spores. Biotechnol. Lett., 29: 1939-1946.

Romero, C. and I. Lopez-Goni, 1999. Improved method for purification of bacterial DNA from bovine milk for detection of Brucella spp. by PCR. Applied Environ. Microbiol., 65: 3735-3737.

Rotz, L.D., A.S. Khan, S.R. Lillibridge, S.M. Ostroff and J.M. Hughes, 2002. Public health assessment of potential biological terrorism agents. Emerg. Infect. Dis., 8: $225-230$.

Sakuma, T., Y. Kurosaki, Y. Fujinami, T. Takizawa and J. Yasuda, 2009. Rapid and simple detection of Clostridium botulinum types $\mathrm{A}$ and $\mathrm{B}$ by loopmediated isothermal amplification. J. Applied Microbiol., 106: 1252-1259.
Tomita, N., Y. Mori, H. Kanda and T. Notomi, 2008. Loop-mediated isothermal amplification (LAMP) of gene sequences and simple visual detection of products. Nat. Protoc., 3: 877-882.

Yamazaki, W., M. Ishibashi, R. Kawahara and K. Inoue, 2008. Development of a loop-mediated Isothermal amplification assay for sensitive and rapid detection of Vibrio parahaemolyticus. BMC Microbiol., 8: 163-163.

Zhu, R.Y., K.X. Zhang, M.Q. Zhao, Y.H. Liu and Y.Y. Xu et al., 2009. Use of visual loop-mediated isotheral amplification of rimM sequence for rapid detection of Mycobacterium tuberculosis and Mycobacterium bovis. J. Microbiol. Methods, 78: 339-343. 